\title{
Does sulphasalazine cause drug induced systemic lupus erythematosus? No effect evident in a prospective randomised trial of 200 rheumatoid patients treated with sulphasalazine or auranofin over five years
}

\author{
Margaret-Mary Gordon, Duncan R Porter, Hilary A Capell
}

\begin{abstract}
Background-Sulphasalazine (SSZ) has been reported to cause drug induced systemic lupus erythematosus (SLE), but diagnosis of this complication in the context of rheumatoid arthritis (RA) is difficult.
\end{abstract}

Objective-To determine prospectively: (1) if patients become seropositive for antinuclear antibodies (ANA) during prolonged treatment with SSZ without clinical evidence of SLE; (2) if ANA positive patients develop more adverse reactions than ANA negative patients; (3) if drug induced SLE was identified in this cohort. Methods-200 patients enrolled in a randomised prospective trial of SSZ and auranofin (AUR) were followed up for five years. Baseline and annual ANA results were collected along with information on drug toxicity and reasons for discontinuation of treatment.

Results-Over five years 24 patients stopped taking SSZ and 49 AUR because of side effects. Of the features common to SLE, rash developed in nine SSZ patients and 11 AUR treated patients and mouth ulcers in three and four patients respectively. Six SSZ treated patients and three treated with AUR developed leucopenia, which promptly resolved with drug withdrawal. No adverse event was ascribed to drug induced SLE. Of the 72 SSZ treated patients who were ANA negative or weakly positive at outset, $14(19 \%)$ became strongly ANA positive compared with 11 (14\%) of 80 AUR patients. Patients ANA positive at baseline or who became ANA positive were not more likely to develop drug toxicity or to withdraw from treatment than those ANA negative throughout.

Conclusion-ANA positivity is common in patients with RA, but the presence or development of ANA did not increase the likelihood of withdrawing from treatment. No case of drug induced SLE was seen over five years in this study. (Ann Rheum Dis 1999;58:288-290)

Gordon, Centre fo

Rheumatic Diseases,

Glasgow Royal Infirmary,

84 Castle Street, Glasgow G4 0SF.

Accepted for publication 26 February 1999

Systemic lupus erythematosus (SLE) is a multifactorial disease. Since the 1970 s, almost 50 drugs have been reported to be associated with drug related lupus. ${ }^{1}$ Hydralazine, penicillamine and procainamide are most commonly implicated, and several case reports have also established sulphasalazine (SSZ) as a causative agent. ${ }^{2-8}$ Diagnosis in the context of rheumatoid arthritis (RA) is difficult as the side effects of several disease modifying drugs (DMARDs) share features of SLE. In addition, "overlap" between RA and SLE is occasionally reported.

Antinuclear antibodies (ANAs) may develop during long term treatment with a number of drugs, and ANAs are invariably detected in those who progress to develop a drug induced lupus syndrome. ${ }^{9-11}$ While the occurrence of antibodies is common, (detected in $50-90 \%$ of patients treated), the frequency of associated disease is rare. The exact incidence of drug induced lupus and of asymptomatic serological abnormalities has not been clearly established. The aim of this study was to determine if treatment with SSZ is associated with: (1) the development of ANA; (2) clinical evidence of drug induced SLE; (3) if there is a relation between ANA status and drug toxicity.

\section{Methods}

Two hundred patients with RA considered to require second line treatment were enrolled in a prospective, randomised trial comparing SSZ and auranofin (AUR) and followed up for five years. One hundred patients were randomised to each group. If the patient had previously been taking SSZ (25 patients) or AUR (four patients), they were allocated to receive the other drug. SSZ was started at $500 \mathrm{mg} /$ day and increased if tolerated to a target dose of 40 $\mathrm{mg} / \mathrm{kg} /$ day. AUR was introduced at $3 \mathrm{mg}$ twice daily, increasing to $3 \mathrm{mg}$ thrice daily if necessary.

Patients were assessed at weeks $0,12,24,48$ and then annually until year 5 . Full blood count, erythrocyte sedimentation rate, $\mathrm{C}$ reactive protein, biochemistry were performed at each visit with measurements of Ritchie articular index, pain score on $10 \mathrm{~cm}$ visual analogue scale and morning stiffness. Baseline and annual ANA results were collected with information on drug toxicity, concomitant medication and reasons for withdrawal from treatment.

\section{Results}

There was no significant difference between the groups with regard to patient details, with a 
Table 1 Proportion of patients continuing treatment over the five years

\begin{tabular}{lllllll}
\hline Year & 0 & 1 & 2 & 3 & 4 & 5 \\
\hline SSZ & 100 & 65 & 56 & 41 & 35 & 31 \\
AUR & 100 & 48 & 38 & 29 & 19 & 15
\end{tabular}

median age of 55 years in the SSZ group and 57 years in the AUR group and median disease duration of 10 years and 9 years respectively. Seventy per cent of patients treated with SSZ were seropositive for rheumatoid factor compared with $75 \%$ of patients treated with AUR. Eighty two per cent of the SSZ treated group and $85 \%$ of the AUR treated group were women. At baseline, patients in the SSZ group had been treated with a mean of one previous DMARD and patients in the AUR group had received an average of 1.1 DMARDs previously. During treatment, only two patients in the SSZ group and one patient in the AUR group received prednisolone $5 \mathrm{mg}$ or $7.5 \mathrm{mg}$ per day. No patients were ever treated with hydralazine or procainamide, which are associated with drug induced lupus. Eighteen of the SSZ group and 24 of the AUR group had previously had penicillamine, but only two of the patients, both in the SSZ group developed a positive ANA.

Thirty four patients had stopped taking SSZ at five years because of lack or loss of effect; 26 patients treated with AUR had also stopped for this reason. There were three non-drug related deaths in the SSZ group and four non-drug deaths in the AUR group. Twenty four patients treated with SSZ and 48 treated with AUR withdrew because of side effects. SSZ was more likely to be continued and to result in a sustained response over the five year period than AUR (table 1). Clinical results have been published more fully by McEntegert et al. ${ }^{12}$

DRUG TOXICITY

Side effects were common, although not all resulted in withdrawal from treatment (table 2). Over five years, 24 patients stopped taking SSZ and 49 AUR because of side effects. The commonest side effects were diarrhoea in the AUR group and rash and nausea in the SSZ group. Six SSZ treated patients and three treated with AUR developed leucopenia, with the lowest total white cell count of 2.2 in one patient treated with SSZ. There were no cases of agranulocytosis. The white cell count promptly returned to the normal range on withdrawing the offending drug.

ANA

At baseline, 72 patients in the SSZ treatment group were ANA negative or weakly positive (titre<1/64). During treatment, 14 (19\%) became strongly ANA positive, at a median of week 24 (range 12-208), but no patients developed clinical features of SLE. In the AUR group, 80 patients were ANA negative or weakly positive at baseline, with 11 (14\%) becoming positive during the treatment period. Patients became ANA positive at a median of week 24 (range 12-96). Again, no patients developed a clinical lupus-like syndrome (table 3).
Table 2 Adverse events over the five years

\begin{tabular}{lll}
\hline & $S S Z$ & AUR \\
\hline Diarrhoea & 2 & 26 \\
Mouth ulcers & 3 & 4 \\
Nausea & 7 & 1 \\
Rash & 9 & 11 \\
Leucopenia & 6 & 3 \\
Thrombocytopenia & 0 & 1 \\
Abnormal LFTs & 2 & 0 \\
Proteinuria & 0 & 3 \\
CNS & 1 & 1 \\
Other & 3 & 6 \\
Total & $33^{\star}$ & $56^{\star}$ \\
\hline
\end{tabular}

${ }^{\star}$ Led to discontinuation of treatment in $24 \mathrm{SSZ}$ and 48 AUR treated patients.

Table 3 Antinuclear antibodies: \% ANA positive at baseline and at time of discontinuation or after five years of treatment

\begin{tabular}{lll}
\hline & $\begin{array}{l}\% \text { ANA positive at } \\
\text { baseline }\end{array}$ & $\begin{array}{l}\% \text { ANA positive after } \\
\text { treatment }\end{array}$ \\
\hline SSZ & 28 & 42 \\
AUR & 20 & 31
\end{tabular}

There was no significant difference between the groups. Patients who were ANA positive at baseline or who became ANA positive during follow up were not more likely to develop drug toxicity or to withdraw from treatment than those who were negative throughout. Antihistone and anti-dsDNA antibodies were not measured in all the patients.

\section{Discussion}

Although the exact aetiology is unclear, SLE is a multifactorial disease in which both genetic and environmental factors, including drug treatment, are involved. Drug induced lupus is a well recognised but uncommon disease entity that occurs with treatment with any of a number of drugs. It differs from idiopathic disease in that malaise and musculoskeletal symptoms predominate, while renal and central nervous system involvement are rare or absent. $^{10}$

The diagnosis of drug related SLE developing on a background of RA may be difficult, as many of the signs, symptoms and serological abnormalities reported in these patients may be a natural consequence of the underlying disease, rather than the drug. This may be particularly true in disease developing in the context of RA treated with SSZ.

SSZ is a commonly used DMARD in RA and other inflammatory joint diseases that has a relatively low risk of toxicity. ${ }^{13}$ RA results in polyarthritis and malaise, and extra-articular features such as pleurisy or pericarditis, or both, are not infrequent manifestations. RA and SLE may rarely coexist but it is more common for ANAs to be found in RA alone in $10-70 \%$ of patients. ${ }^{14} 15$ The development of ANAs or dsDNA antibodies should not necessitate withdrawal from treatment, although careful follow up will be required. These antibodies develop in $5-15 \%$ of patients taking SSZ for RA, without clinical symptoms of SLE. ${ }^{16}$ The most useful diagnostic criteria for drug induced SLE is the rapid improvement of the clinical features after withdrawal of the drug, thus providing evidence that the lupus 
syndrome is induced by SSZ rather than unmasked by it. Failure to recognise this complication may lead to delay in withdrawing the offending drug or inappropriate treatment.

In one review of SSZ induced SLE, $88 \%$ had arthralgia, 33\% had fever and 25\% had rashes. ${ }^{17}{ }^{18}$ Leucopenia, anaemia, vasculitis and pneumonitis have also been reported although serious renal disease has not been reported. ${ }^{2}$ ANAs often develop, but dsDNA antibodies, which are highly specific for SLE, are rarely found in drug induced lupus. ${ }^{19}$

Serological abnormalities are often found, but the frequency of drug induced lupus is low. ANAs may be detected in as many as $50-90 \%$ of patients depending on the drug, dose and duration of treatment. ${ }^{9}$ The development of ANAs suggests a drug induced autoimmune phenomenon, resulting in other antibodies than the classic antihistones. The true incidence and prevalence has never been clearly elucidated, but it remains that more patients develop ANAs but remain asymptomatic than develop clinical symptoms.

SSZ is reduced in the colon to 5-aminosalicylic acid and sulphapyridine. 5-ASA is mostly unabsorbed and sulphapyridine is acetylated. Genetically determined variation in acetylation leads to variation in rates of metabolism. Sixty per cent of the British population are slow acetylators and are more likely than rapid acetylators to experience adverse reactions with drugs requiring acetylation for metabolism and are also more likely to develop drug induced SLE. ${ }^{20}$

Although of moderate size and duration, this study is still too small and follow up too short to provide answers about uncommon side effects from either SSZ or AUR. Baseline ANA status should be determined before treatment with any of the drugs known to be incriminated in the development of drug induced lupus and patients should be observed carefully for signs and symptoms of SLE. Indeed, as so many drugs have been implicated to date, all patients starting DMARD treatment for inflammatory arthritis should have baseline ANA checked.

In conclusion, ANAs are common in patients with RA. Side effects of commonly used DMARDs share features with signs and symptoms of SLE. In this study, $11-14 \%$ of patients became strongly ANF positive during follow up, but the presence or development of ANAs did not correlate with clinical disease or increase the likelihood of withdrawing from treatment. Larger cohorts followed up prospectively are being studied to elucidate the relevance of this effect in long term use of SSZ.

We would like to thank Professor R D Sturrock and Drs J Hunter, R Madhok and M Field for permission to study their patients. We are also grateful to Mrs M Morrison and Mrs E A Thomson for metrology help.

Funding: none.

Conflicts of interest: none.

1 Hess E. Drug-related lupus. N Engl J Med $1988 ; 318: 1460-3$.

2 Clementz GL, Dolin BJ. Sulfasalazine-induced lupus erythematosus. Am J Med 1988;84:535-8.

3 Laversuch CJ, Collins DA, Charles PJ, Bourke BE Sulphasalazine-induced autoimmune abnormalities in patients with rheumatic disease. Br J Rheumatol 1995;34: 435-9.

4 Caulier M, Dromer C, Andrieu V, Le Guennec P, Fournie B. Sulphasalazine-induced lupus in rheumatoid arthritis. J Rheumatol 1994;21:750-1.

5 Carr-Locke DL. Sulfasalazine-induced lupus syndrome in a patient with Crohn's disease. Am J Gastroenterol 1983;77: 614-16.

6 Griffiths ID, Kane SP. Sulphasalazine-induced lupus syndrome in ulcerative colitis. BMJ 1997;2:188-9.

7 Deboever G, Devogelaere R, Holvoet G. Sulphasalazineinduced lupus like syndrome with cardiac tamponade in a patient with ulcerative colitis. Am J Gastroenterol 1988;84: p5-6.

8 Veale DJ, Ho M, Morley KD. Sulphasalazine-induced lupus in a patient with psoriatic arthritis. $\mathrm{Br} \mathrm{J}$ Rheumato $1995 ; 34: 383-4$

9 Hobbs RN, Clayton AL, Bernstein RM. Antibodies to the five histones and poly (adenosine diphosphate-ribose) in drug induced lupus: implications for pathogenesis. Ann Rheum Dis 1987;46:408-16.

10 Skaer TL. Medication-induced systemic lupus erythematosus. Clin Ther 1992;14:496-506.

11 Stratton MA. Drug induced systemic lupus erythematosus. Clinical Pharmacy 1985;4:657-63.

12 Mc Entegert A, Porter D, Capell HA, Thomson EA. Sulfasalazine has a better efficacy/ toxicity profile than auranofin: evidence from a 5 year prospective, randomized auranofin: evidence from a 5 year prosp

13 Porter DR, McInnes I, Hunter J, Capell HA. Outcome of second line therapy in rheumatoid arthritis. Ann Rheum Dis 1994:53:812-15.

14 Barnett EV, North AE, Condemi JJ. Anti nuclear factors in systemic lupus erythematosus and rheumatoid arthritis. Ann Intern Med 1965;63:100-8.

15 Linn JE, Hardin JG, Halla JT. A controlled study of ANA+ and RF- arthritis. Arthritis Rheum 1978;21:645-51.

16 Caulier M, Dromer C, Andrieu V, Le Guennec P, Fournie B. Sulfasalazine induced lupus in rheumatoid arthritis. J Rheumatol 1994;21:750-1.

17 Alarcon-Segovia D, Kraus A. Drug related lupus syndromes and their relationship to spontaneously occurring systemic lupus erythematosus. Ballieres Clin Rheumatol 1991;5:112 .

18 Mongey AB, Hess EV. Sulphasalazine-induced systemic lupus erythematosus. Br J Rheumatol 1994;33:789-90.

19 Mielke H, Wildhangen K, Mau W, Ziedler H. Follow up of patients with double stranded DNA antibodies induced by sulfasalazine during the treatment of inflammatory rheumatic diseases. Scand J Rheumatol 1993;22:299-301.

20 Drayer DE, Reidenberg MM. Clinical consequences of polymorphic acetylation of basic drugs. Clin Pharmacol Ther $1977 ; 22: 251-8$ 